\title{
Learning curves for endobronchial ultrasound using cusum analysis
}

\author{
S V Kemp, ${ }^{1}$ S H El Batrawy, ${ }^{1}$ R N Harrison, ${ }^{2}$ K Skwarski, ${ }^{3}$ M Munavvar, ${ }^{4}$ A Roselli, ${ }^{5}$ \\ K Cusworth, ${ }^{2} \mathrm{PL}$ Shah $^{1}$
}

${ }^{1}$ Department of Respiratory Medicine, Royal Brompton Hospital, London, UK ${ }^{2}$ Department of Respiratory Medicine, University Hospital of North Tees, Stockton-on-Tees, UK

${ }^{3}$ Respiratory Medicine Unit, Royal Infirmary of Edinburgh, Edinburgh, UK

${ }^{4}$ Department of Respiratory Medicine, Royal Preston Hospital, Preston, UK ${ }^{5}$ Department of Respiratory Medicine, Barcelona, Spain

\section{Correspondence to}

Samuel Kemp, Department of Respiratory Medicine, Royal Brompton Hospital, Fulham Road, London SW3 6NP, UK; s.kemp@rbht.nhs.uk

Received 13 September 2009 Accepted 13 March 2010

\begin{abstract}
Background The assessment of medical trainees is becoming an increasingly prominent issue, with current methods having varying degrees of inherent subjectivity and bias. Cusum analysis is a technique used in quality control systems, and is starting to be employed in medical training. Endobronchial ultrasound (EBUS) is an established tool in the diagnosis and staging of lung cancer, although its use in the UK is currently restricted. As it becomes more widespread, there will be a need to assess trainees' competence accurately to ensure that those performing EBUS at new centres are appropriately skilled.
\end{abstract}

Methods A retrospective review of clinical practice in tertiary referral centres in England, Scotland and Spain was carried out. The study group comprised 500 patients undergoing EBUS for the diagnosis and staging of lung cancer as part of a clinical service. Using cusum analysis, the first 100 cases from each of the five centres are presented. Each centre has one consultant physician as the primary EBUS operator, and all operators began using EBUS at their current centre (ie, no learning from prior experience). The data are presented as learning curves. Results It is evident that there is a wide range of time over which EBUS-guided transbronchial needle aspiration (TBNA) competence is attained. The pooled sensitivity was $67.4 \%$ (individual sensitivities $66.7,70.7,61.2,80.3$ and $59.7 \%$ ).

Conclusion Cusum analysis is well suited to the assessment of procedures with a binary outcome, but accurate and appropriate standards of practice must be determined prior to assessment to ensure correct identification of underperformance. This report suggests that the learning curve for EBUS is greater than previously reported using different methods, and that even experienced bronchoscopists vary in their speed of learning.

\section{INTRODUCTION}

There has been much discussion over the years about how best to assess the competence of doctors to perform a given task or procedure. The National Health Service (NHS) spent $>£ 550$ million on negligence claims (including defence costs, damages and claimants legal fees) in the 2007-2008 financial year, ${ }^{1}$ highlighting the need to ensure appropriate and rigorous training of all healthcare professionals. There has been a move away from the 'see one, do one, teach one' philosophy of old where there was an assumption of competence, to more structured assessments of trainees' performances. In many centres, competence is assessed with the use of logbooks, with an arbitrary number of any given procedure being taken as a sign of competence. This clearly does not account for the different rates at which people learn, leaving some trainees frustrated at overly heavy supervision late in their training while risking cutting others adrift too early. A good analogy would be learning to drive. Some people require little time and few lessons, while others spend months taking lessons and failing tests before finally succeeding. The introduction of documentation such as 'Directly Observed Procedures' (DOPs) has gone some way to correct this, but there are still inherent problems with such systems. The trainee is in control of which procedures are actually assessed, and by whom, so that there is a strong bias to successful procedures assessed by friendly or lenient colleagues. This gives a false sense of security in the ability of any given trainee at end-of-year formal assessments, but can also (and more concerningly) lead to overconfidence on the part of that trainee who may develop over time a skewed perception of his or her own performance.

Cusum analysis is a method of continuously assessing the performance of an individual or process against a predetermined standard in order to detect adverse trends and to allow for early intervention (eg, re-training). First described by Page in his 1954 paper on continuous inspection schemes, ${ }^{2}$ it has been widely adapted by the manufacturing and energy industries as an important tool in quality control processes. Recently, several papers have appeared looking at the use of cusum analysis in a variety of clinical scenarios, predominantly in the fields of surgery and anaesthesia (ie, procedure-heavy specialities). ${ }^{3-7}$

In this report, we have applied cusum analysis to the learning of endobronchial ultrasound-guided transbronchial needle aspiration (EBUS TBNA) of mediastinal lymph nodes in the investigation of suspected lung cancer. EBUS TBNA is becoming an increasingly utilised technique for the diagnosis and staging of lung cancer, with two meta-analyses published recently reporting high sensitivities and specificities. The first, a meta-analysis of 11 studies with a total of 1299 patients reported a pooled sensitivity of 0.93 (95\% CI 0.91 to 0.94$)$ and specificity of $1.00(95 \% \mathrm{CI} 0.99$ to 1.00$),{ }^{8}$ and the second, with 10 studies and 817patients, reported a pooled sensitivity of 0.88 ( $95 \%$ CI 0.79 to 0.94 ) and pooled specificity of $1.00(95 \%$ CI 0.92 to 1.00). ${ }^{9}$ It is cheaper than mediastinoscopy, can be performed as a day-case procedure, and morbidity is extremely low. In the UK, operators are largely selftaught, as there are currently too few centres with an established EBUS service to provide training to 
more than a handful of junior doctors. This has presented a unique opportunity to assess the learning of a new skill without confounders such as supervisor intervention. We report the learning curves of five independent EBUS TBNA operators by retrospectively applying cusum analysis to the first 100 cases of each. The data represent the use of EBUS TBNA in clinical practice, rather than in the more controlled environment of a clinical trial, and the retrospective analysis in this series has prevented case selection and non-routine practices that may be used when the operator is aware that he is being monitored.

\section{METHODS}

Each of the five centres involved submitted a database of their first 100 EBUS TBNA cases. Each centre had one consultant respiratory physician who was the principle operator in each and every procedure, with help administered where necessary by trainees or bronchoscopy nursing staff (eg, maintaining scope position at the mouth during needle advancement). A procedure was defined as the patient being booked for EBUS TBNA, signing the consent form and keeping their appointment time. Any reason for the procedure subsequently not going ahead or being abandoned early was documented as a procedural failure. All procedures were carried out under conscious sedation. Information was collected on the size and station of lymph nodes targeted, results of preprocedure positron emission tomography (PET) scans where performed, cytology results from TBNA samples, final diagnosis, how that diagnosis was reached if not by EBUS TBNA, and any resulting complications. To avoid inconsistencies in outcome assignment, the results from all five centres were analysed by the same panel of three assessors (SK, SE and PS), and each result was designated as either true or false. Designations are defined below, and any case in which there was incomplete information or doubt about outcome was assigned as a false negative, so as to err on the side of caution.

True positive

TBNA cytology diagnostic of malignancy (or other process). True negative

TBNA cytology negative and subsequent investigations confirmed a non-cancer diagnosis

or

TBNA cytology negative with inconclusive or no subsequent investigations and patient alive and well at 1 year and original abnormalities ascribed to a non-cancer process.

False negative

TBNA cytology negative but subsequent investigations confirm a diagnosis of cancer

or

TBNA cytology negative with inconclusive or no subsequent investigations and progressive disease clinically ascribed to cancer

or

TBNA cytology negative with inconclusive or no subsequent investigations but patient not alive at 1 year with death ascribed to cancer

or

TBNA cytology negative with inconclusive or no subsequent investigations and patient lost to follow-up

or

Procedural failure.

False positive

TBNA cytology positive for malignancy but histopathology at further biopsy/resection/postmortem showed different malignancy.
Cusum analysis was applied to produce a learning curve for each centre. A good summary of cusum analysis has been published by Bolsin and Colson, ${ }^{10}$ and the following outline is based on their explanations.

An outcome score $\mathbf{X}$ is allocated to each procedure, where $\mathbf{X}_{\mathbf{n}}$ is the outcome score of procedure $n$. The reward for a successful procedure (s) is usually less than the penalty for a failed procedure $(\mathbf{1}-\mathbf{s})$, such that $>1$ success is needed to redress the balance following a failure. To determine the value of $\mathbf{s}$, one must set an acceptable failure rate, $\mathbf{p}_{\mathbf{0}}$ (the level of inherent error if the procedure is carried out correctly), and an unacceptable failure rate, $\mathbf{p}_{\mathbf{1}}$ (where $\mathbf{p}_{\mathbf{1}}-\mathbf{p}_{\mathbf{0}}$ represents the maximum acceptable level of human error) for the particular procedure being assessed, where:

$$
\mathbf{P}=\ln \left(\mathrm{p}_{1} / \mathrm{p}_{0}\right) \quad \mathbf{Q}=\ln \left(\left(1-\mathrm{p}_{1}\right) /\left(1-\mathrm{p}_{0}\right)\right) \quad \mathrm{s}=\mathrm{Q} /(\mathrm{P}+\mathrm{Q})
$$

In the case of EBUS TBNA, we have designated acceptable and unacceptable failure rates as $10 \%$ and $20 \%$, respectively. Thus:

$$
\mathbf{p}_{0}=0.1 \quad \mathbf{p}_{1}=0.2 \quad \mathbf{s}=0.15 \quad \mathbf{1}-\mathbf{s}=0.85
$$

A cusum score $\mathbf{C}_{\mathbf{n}}$ can then be generated. At the start of the period of analysis, $\mathbf{C}_{\mathbf{0}}=0$, and $\mathbf{C}_{\mathbf{n}}$ is the sum of all individual outcome scores $\left(\mathbf{X}_{\mathbf{1}}, \mathbf{X}_{\mathbf{2}}, \ldots, \mathbf{X}_{\mathbf{n}}\right)$. An upward projection of the graphed cusum score suggests a success rate below that expected, and a stable or downward projection indicates competence.

The cusum graph is said to signal when $\mathbf{C}_{\mathbf{n}}$ crosses a predetermined decision interval, $\mathbf{H}$. $\mathbf{H}_{\mathbf{0}}$ denotes the value between each acceptable decision interval, and $\mathbf{H}_{\mathbf{1}}$ the value between each unacceptable decision level. These intervals can be marked as horizontal lines on the cusum graph. When a line is crossed, $\mathbf{C}_{\mathbf{n}}$ traditionally reverts to 0 , but a learning curve can be constructed if repeated decision intervals are stacked graphically. In order to determine $\mathbf{H}$, we need to know the type 1 error (odds of falsely accusing an operator of being incompetent, designated $\alpha$ below) and type 2 error (odds of falsely certifying someone as competent, designated $\beta$ below). For a training programme, one would ideally like to set a relatively high type 1 error and a low type 2 error to ensure that as few people as possible slip through the net. However, in order to produce an easily interpretable graph, it is best to give both type 1 and 2 errors the same value, as this allows both acceptable and unacceptable performance decision intervals to be equal. We have given a value of 0.1 to both for this analysis.

$$
\begin{aligned}
& \mathbf{a}=\ln ((1-\beta) / \boldsymbol{\alpha}) \quad \mathbf{b}=\ln ((1-\boldsymbol{\alpha}) / \beta) \quad \mathbf{H}_{0}=\mathrm{b} /(\mathrm{P}+\mathrm{Q}) \\
& \mathbf{H}_{1}=\mathrm{a} /(\mathrm{P}+\mathrm{Q})
\end{aligned}
$$

Thus in our series:

$$
\mathbf{H}_{0}=2.71 \quad \mathbf{H}_{1}=2.71
$$

\section{RESULTS}

Results are presented graphically as learning curves (figure 1). A positive deflection indicates a false result obtained at any given procedure, and a negative deflection represents a true result.

It is evident immediately from the graphical data that there is a range of time over which EBUS TBNA competence is attained. Operator 1 has a very steep learning curve initially, which starts to level off after the 32 nd procedure. The last new unacceptable decision interval crossed occurs at the 56th procedure, after which the cusum curve flattens before taking a consistently downward path. Operator 2 seems to perform well initially, but then the cusum curve turns upwards until crossing a decision 
Figure 1 Cusum curves by operator.
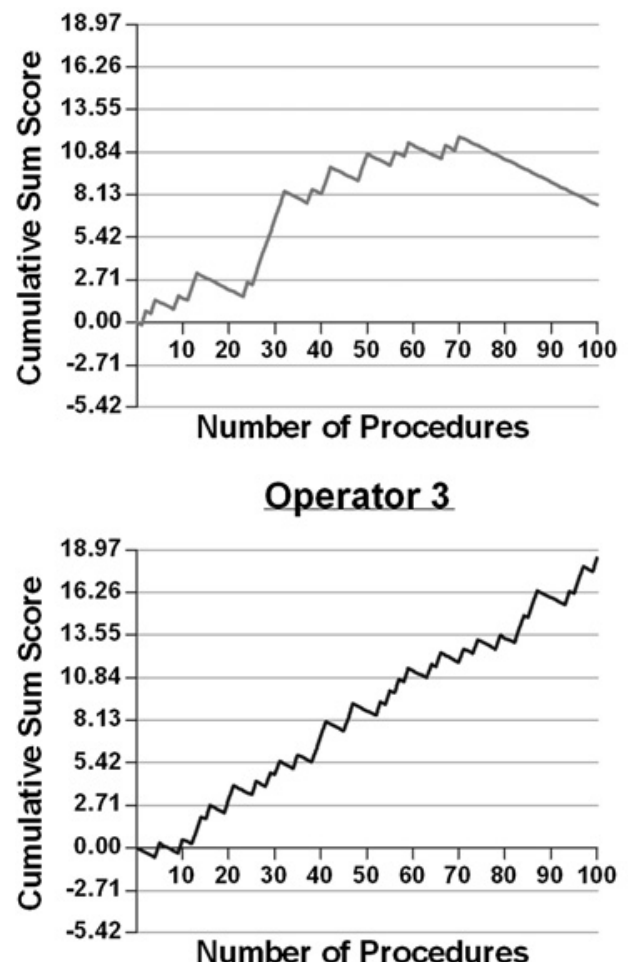

Operator 2
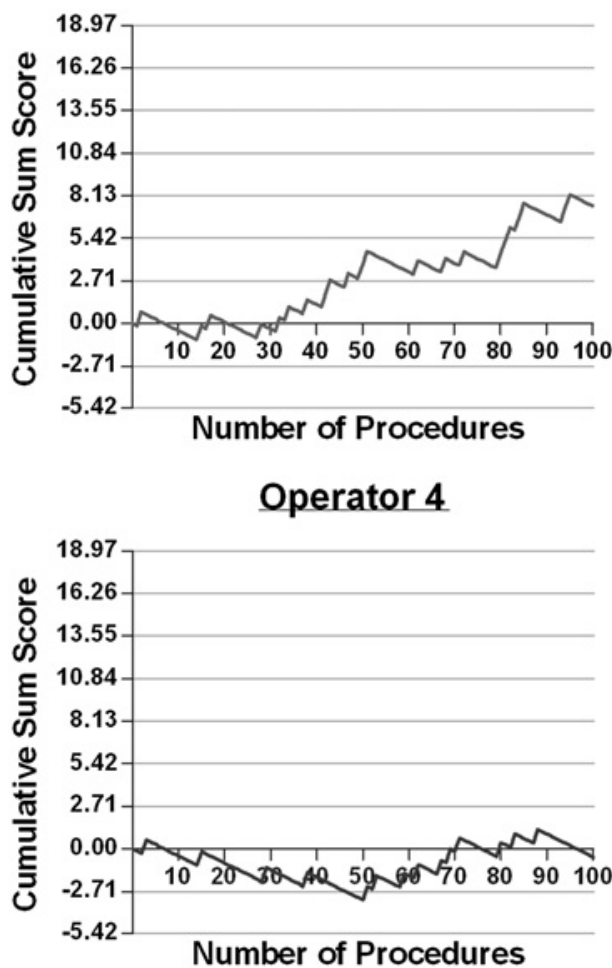

\section{Operator 5}

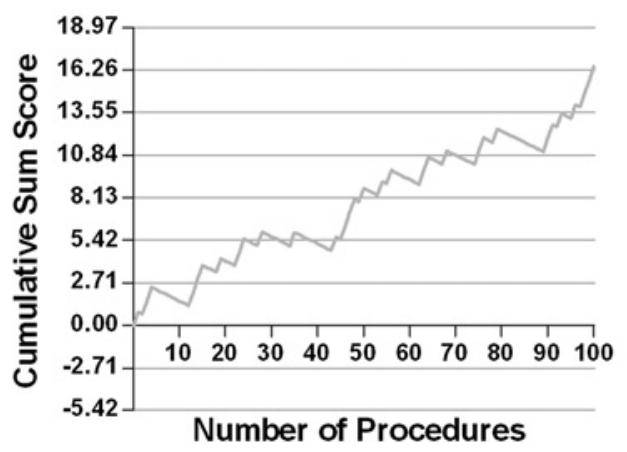

interval at the 43rd procedure. This is followed by another period of stability, before the curve takes another step upwards at around the 80 th procedure. The curve has largely levelled out by the end of the series. The cusum curves of operators 3 and 5 both show a relentless rise over the 100 procedures, indicating that they are still in the learning phase. Operator 4 appears to have attained competence almost immediately, and the cusum curve essentially remains flat for the duration of the analysis period.

The data give a pooled sensitivity of $67.4 \%$ (95\% CI $51.5 \%$ to $84.0 \%$; individual sensitivities $66.7,70.7,61.2,80.3$ and $59.7 \%$ ). Positive cytology at EBUS TBNA is rarely followed by confirmatory surgical biopsies in clinical practice, and therefore it is not possible to assess specificity accurately. However, in the few cases in this series in which additional lymph node tissue was obtained (eg, in those with positive N1 nodes), there were no false-positive results.

\section{DISCUSSION}

At present, many assessments are seen by trainees as time consuming and of limited usefulness, and by assessors as simply more paperwork. Finding a useful, appropriate and continuous measure of competence is essential to engage everyone in trainee assessment. This is likely to lead to benefits for both sides-correct and prompt (re-)training for junior staff, and the reassurance for managers and consultants that those juniors are correctly trained. There would thus be an onus on institutions to ensure that adequate training was made available for those learning new procedures, and to provide the necessary tools for continuous assessment. These measures would help in negligence claims, as it would be easy for institutions to demonstrate that staff were competent and that sufficient training was available to allow staff to attain that competence.

Table 1 Number of nodes $<1 \mathrm{~cm}$ sampled by operator

\begin{tabular}{lll}
\hline Operator & No. of nodes & No. of patients \\
\hline 1 & 19 & 10 \\
2 & 1 & 1 \\
3 & 1 & 1 \\
4 & 3 & 3 \\
5 & 17 & 17 \\
\hline
\end{tabular}


The results from the meta-analyses discussed in the Introduction contrast markedly with the data reported here, with a pooled sensitivity of $67.4 \%$. The discrepancy between our data and the meta-analyses probably reflects two things. First, that these cases constituted usual clinical practice, rather than a controlled prospectively monitored cohort as may have been the case in many series, and secondly that the case series used in the meta-analyses were performed by experienced operators rather than by novices. Furthermore, sensitivity is strongly influenced by prevalence, and studies where there is a higher prevalence of cancer are likely to generate a higher sensitivity. The sensitivity was also reduced in the data that we have presented as several cases have been assigned a false-negative outcome on the basis of uncertainty or loss to follow-up.

One possible reason for the differing learning curves could be the proportion of large versus small $(<10 \mathrm{~mm})$ nodes sampled. From the available data, the number of patients in which exclusively subcentimetre nodes were sampled by each of operators $1-5$ is shown in table 1 . Similarly, the length of time taken to perform 100 cases varied greatly, from 12 months and 4 days (operator 4) to 51 months and 24 days (operator 1). The slow initial accrual of cases by operator 1 could certainly have contributed to the continued upward trend of the cusum curve for the first 50 or so procedures. Recruitment time for the first 50 cases was 28 months and 21 days, and for the second 50 cases was 13 months and 3 days. Operator 4 was performing two procedures a week throughout the data collection period, leading to the quickest case accrual and the best overall cusum curve.

We set acceptable and unacceptable failure rates at $10 \%$ and $20 \%$, respectively, based on 'expert opinion' prior to any analysis of data. These values fit well with the meta-analyses by Gu et al and Adams et al, ${ }^{8}$ but, with the comments above in mind, these may be considered very tough standards. It may be that these rates could be changed depending upon experience. For example, if we drop the acceptable and unacceptable failure rates to $15 \%$ and $25 \%$, respectively, we see that operator 2 , like operator 4, now appears to have achieved and sustained competence immediately, and operator 1 achieves competence 15 procedures earlier (41 vs 56 procedures). Results are shown graphically in figure 2 .
Figure 2 Cusum curves with less harsh failure criteria.
Operator 1
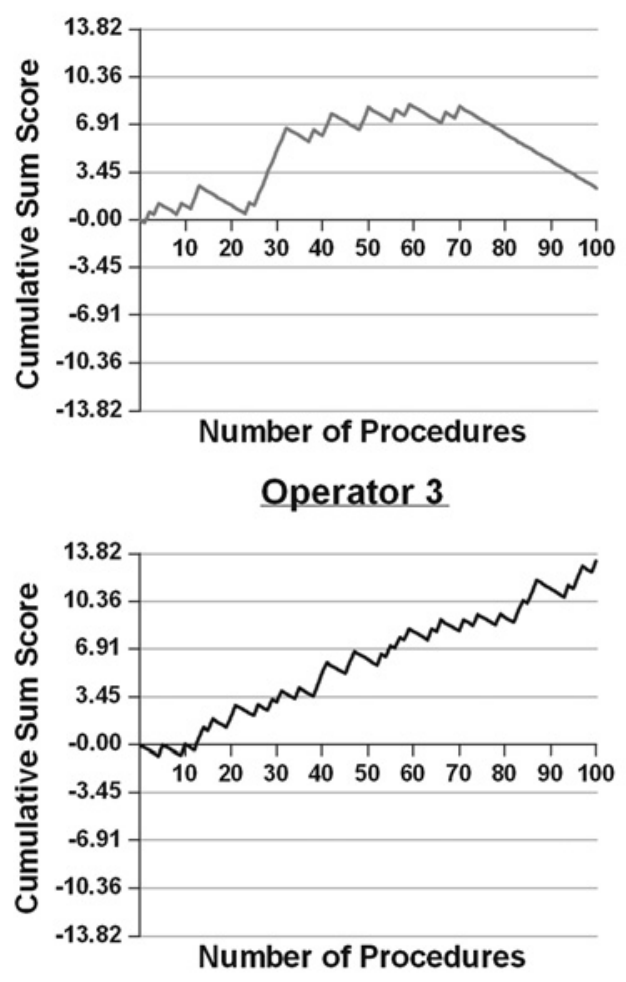

Operator 2
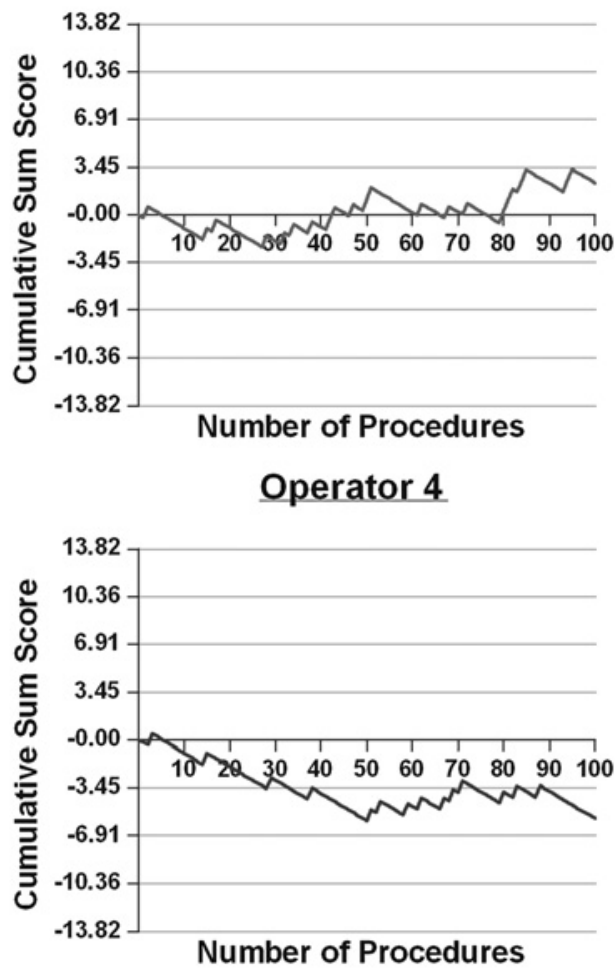

Operator 5

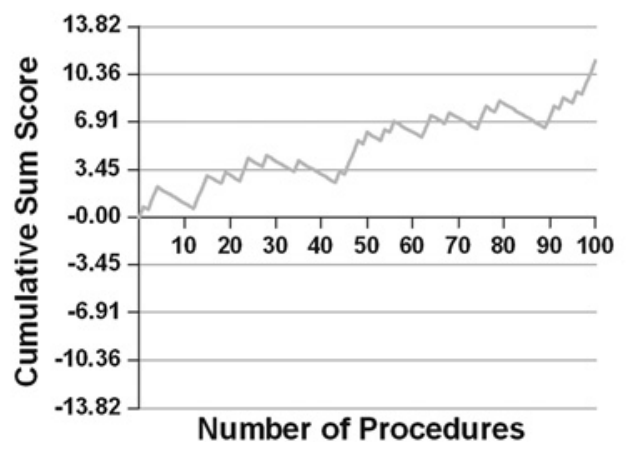




\section{What is already known on this subject}

Trainee assessment techniques are constantly evolving.

- There is an increasing emphasis on proving on-going competence.

- Cusum analysis is an emerging assessment tool in medicine.

\section{What this study adds}

- Emphasises variability in learning.

- Provides a potential model for evaluating competence in both trainees and consultants.

- Provides model for monitoring ongoing practice.

There are currently no published guidelines in the UK for what constitutes adequate training in bronchoscopy, or how best to assess competence. The data presented here clearly demonstrate different speeds at which people learn new tasks, even when we consider operators highly experienced in similar procedural techniques. With the ever-expanding number and complexity of bronchoscopic procedures (and of medical procedures in general), there is a need to formulate adequate assess- ment tools for trainee development, and we propose cusum analysis as one such tool. However, accurate and appropriate standards of practice must be determined prior to assessment to ensure correct identification of those who are underperforming.

Competing interests None.

Provenance and peer review Not commissioned; externally peer reviewed.

\section{REFERENCES}

1. Hansard (House of Commons Daily Debates) Written Answers 6th May 2008.

2. Page ES. Continuous inspection schemes. Biometrika 1954;41:100-15.

3. Naik VN, Devito I, Halpern SH. Cusum analysis is a useful tool to assess resident proficiency at insertion of labour epidurals. Can J Anaesth 2003;50:694-8.

4. Runcie C. Cusum analysis can monitor the practical skills of a consultant cardiac anaesthetist. Anaesthesia 2008;63:908-9.

5. Colquhoun PH. CUSUM analysis of J-pouch surgery reflects no learning curve after board certification. Can J Surg 2008;51:296-9.

6. Toker A, Tanju S, Ziyade S, et al. Learning curve in videothoracoscopic thymectomy: how many operations and in which situations? Eur J Cardiothorac Surg 2008;34:155-8.

7. East JM, Valentine CS, Kanchev E, et al. Sentinel lymph node biopsy for breast cancer using methylene blue dye manifests a short learning curve among experienced surgeons: a prospective tabular cumulative sum (CUSUM) analysis. BMC Surg 2009;9:2

8. Gu P, Zhao YZ, Jiang LY, et al. Endobronchial ultrasound-guided transbronchial needle aspiration for staging of lung cancer: a systematic review and meta-analysis. Eur J Cancer 2009;45:1389-96.

9. Adams K, Shah P, Edmonds L, et al. Endobronchial ultrasound and transbronchial needle aspiration biopsy for mediastinal staging in patients with lung cancer: systematic review and meta-analysis. Thorax 2009;64:757-62.

10. Bolsin S, Colson M. The use of the cusum technique in the assessment of trainee competence in new procedures. Int J Oual Health Care 2000;12:433-8.

\section{Lung alert}

\section{MMP12 and reduced risk of COPD}

Not all smokers develop chronic obstructive pulmonary disease (COPD), and not everyone with COPD has the same rate of decline of lung function. In this study the authors hypothesised that matrix metalloproteinase 12 (MMP12) variants have a role in lung function and are risk factors for COPD. MMP12 is produced by macrophages, one of the main inflammatory cells associated with smoking.

Seven different cohorts that included two cohorts of children with asthma, one birth cohort and two cohorts of adults with and without COPD were tested for an association between single nucleotide polymorphisms (SNPs) in the MMP12 gene and force expiratory volume in $1 \mathrm{~s}\left(\mathrm{FEV}_{1}\right)$.

The minor allele of a functional variant of MMP12 was shown to have a positive association with $\mathrm{FEV}_{1}$ in children with asthma and in adult smokers with COPD or at risk of COPD. The expression of this allele had a beneficial effect on lung function and was associated with a reduced risk of COPD in adult smokers.

These findings lend weight to the 'Dutch hypothesis' of asthma and COPD. The fact that the cohorts were diverse and many of them were on various medications may explain why the association was significant in combined analyses of the cohorts but not so in the individual cohorts. Nevertheless this study adds more weight to the importance of a genetic element in the development of COPD.

Hunninghake GM, Cho MH,Tesfaigzi Y, et al. MMP12, lung function, and COPD in high-risk populations. N Engl J Med 2009;361:2599-608.

\section{A Pillai}

North Bristol Lung Centre, Bristol

Correspondence to Anilkumar Pillai, Gloucestershire Royal Hospital, UK; anilkumarpillai@doctors.org.uk Thorax 2010;65:538. doi:10.1136/thx.2010.141085 


\section{REFERENCES}

1. Anderson SD. Exercise-induced asthma. In: Kay AB, ed. Allergy and allergic diseases. Oxford: Blackwell Scientific Publications, 1997:672-711.

2. Rundell KW, Im J, Mayers LB, et al. Self-reported symptoms and exercise-induced asthma in the elite athlete. Med Sci Sports Exerc 2001; 33:208-13.

3. Dickinson JW, Whyte GP, McConnell AK, et al. Midexpiratory flow versus FEV1 measurements in the diagnosis of exercise induced asthma in elite athletes. Thorax 2006;61:111-4.

4. Holzer K, Douglass JA. Exercise induced bronchoconstriction in elite athletes: measuring the fall. Thorax 2006;61:94-6.

5. Parsons JP, Mastronarde JG. Exercise-induced bronchoconstriction in athletes. Chest 2005;128:3966-74.

\section{Corrections}

doi:10.1136/thx.2009.122291 corr1

Conway Morris A, Kefala K, Wilkinson $\mathrm{TS}$, et al. Diagnostic importance of pulmonary interleukin-1b and interleukin-8 in ventilator-associated pneumonia. Thorax 2010;65:201-7. This article should have included the note that Dr Kefala was joint first author.
Polverino E, Dambrava P, Cilloniz C, et al. Nursing home-acquired pneumonia: a 10 year single-centre experience. Thorax 2010;65:354-59. The correct affiliation for affiliation 1 should have read "Respiratory Department, Hospital Clinic-IDIBAPS, Barcelona-Spain, Centro de Investigación Biomedica En RedEnfermedades Respiratorias (CibeRes, CB06/06/0028, el Ciberes es una iniciativa del ISCIII) - 2009SGRO http://www.idibapsrespiratoryresearch. org."

doi:10.1136/thx.2009.133108corr1

Millett C, Glantz SA. Assigning an '18' rating to movies with tobacco imagery is essential to reduce youth smoking. Thorax 2010;65:377-8. The authors referred to a paper by McNeil et al; this should have been Lyons et al (Lyons A, McNeill A, Chen Y, et al).
Lyons A, McNeill A, Chen Y, et al. Tobacco and tobacco branding in films most popular in the UK from 1989 to 2008. Thorax 2010;65:417-22. There is an error in figure legend 2 which currently reads "Trends in all tobacco intervals and tobacco use intervals per hour per day by British Board of Film Classification (BBFC) category (all figures expressed as means)." It should have read: "Trends in all tobacco intervals and tobacco use intervals per hour per year by British Board of Film Classification (BBFC) category (all figures expressed as means)."

doi:10.1136/thx.2009.127274corr1

Kemp SV, El Batrawy SH, Harrison RN, et al. Learning curves for endobronchial ultrasound using cusum analysis. Thorax 2010;65:534-8. The author name A Roselli should have read A Rosell. 
conditioning replacement has probably been overestimated because the emergence of the new 19A serotype has also been observed when the vaccine was not used. ${ }^{6}$ Moreover, the importance of PCV in reducing the incidence of pneumococcal community-acquired pneumonia by about $23 \%$ in a low-income, low-mortality developing Asian country, the Philippines, has recently been clearly demonstrated by Lucero et al. ${ }^{7}$

Finally, the use of Hib vaccines and PCV can induce significant herd immunity, thus justifying the conclusion that both should used in Asian children.

\section{Nicola Principi, Susanna Esposito}

Department of Maternal and Pediatric Sciences, Università degli Studi di Milano, Fondazione IRCCS Ca' Granda Ospedale Maggiore Policlinico, Milan, Italy

Correspondence to Nicola Principi, Department of Maternal and Pediatric Sciences, Università degli Studi di Milano, Fondazione IRCCS "Ospedale Maggiore Policlinico, Mangiagalli e Regina Elena", Via Commenda 9, 20122 Milano, Italy; nicola.principi@unimi.it

Funding Bando Giovani Ricercatori 2007, Italian Ministry of Health.

\section{Competing interests None.}

Provenance and peer review Not commissioned; not externally peer reviewed.

Accepted 26 January 2011

Published Online First 17 April 2011

Thorax 2012;67:83-84.

doi:10.1136/thx.2011.160135

\section{REFERENCES}

1. Das RR. Childhood immunisation with conjugate vaccines and prevention of pneumonia. Thorax 2012;67:85

2. Principi N, Esposito S. Management of severe community-acquired pneumonia of children in developing and developed countries. Thorax 2011;66:815-22.

3. Eskola J. Foresight in medicine: current challenges with Haemophilus influenzae type $\mathrm{b}$ conjugate vaccines. J Intern Med 2010;267:241-50.

4. Theodoratou $\mathbf{E}$, Johnson S, Jhass A, et al. The effect of Haemophilus influenzae type $b$ and pneumococcal conjugate vaccines on childhood pneumonia incidence, severe morbidity and mortality. Int $J$ Epidemiol 2010:39(Suppl 1):i172-85.

5. Gessner BD. Haemophilus influenzae type b vaccine impact in resource-poor settings in Asia and Africa. Expert Rev Vaccines 2009;8:91-102.
6. Choi EH, Kim SH, Eun BW, et al. Streptococcus pneumoniae serotype 19A in children, South Korea. Emerg Infect Dis 2008;14:275-81.

7. Lucero MG, Nohynek H, Williams G, et al. Efficacy of an 11-valent pneumococcal conjugate vaccine against radiologically confirmed pneumonia among children less than 2 years of age in the Philippines: a randomized, double-blind, placebo-controlled trial. Pediatr Infect Dis J 2009;28:455-62.

\section{CORRECTION}

doi:10.1136/thx.2009.127274corr2

Kemp SV, El Batrawy SH, Harrison RN, et al. Learning curves for endobronchial ultrasound using cusum analysis. Thorax 2010;65:534-8. doi:10.1136/thx.2009. 127274. This paper has a formula incorrectly specified. The formula for $\mathrm{Q}$ on page 535 which reads $\mathrm{Q}=\ln \left(\left(1-\mathrm{p}_{1}\right) /\left(1-\mathrm{p}_{0}\right)\right)$ should read: $Q=\ln \left(\left(1-p_{0}\right) /\left(1-p_{1}\right)\right){ }^{2}$

\section{SAVE TIME AND KEEP INFORMED}
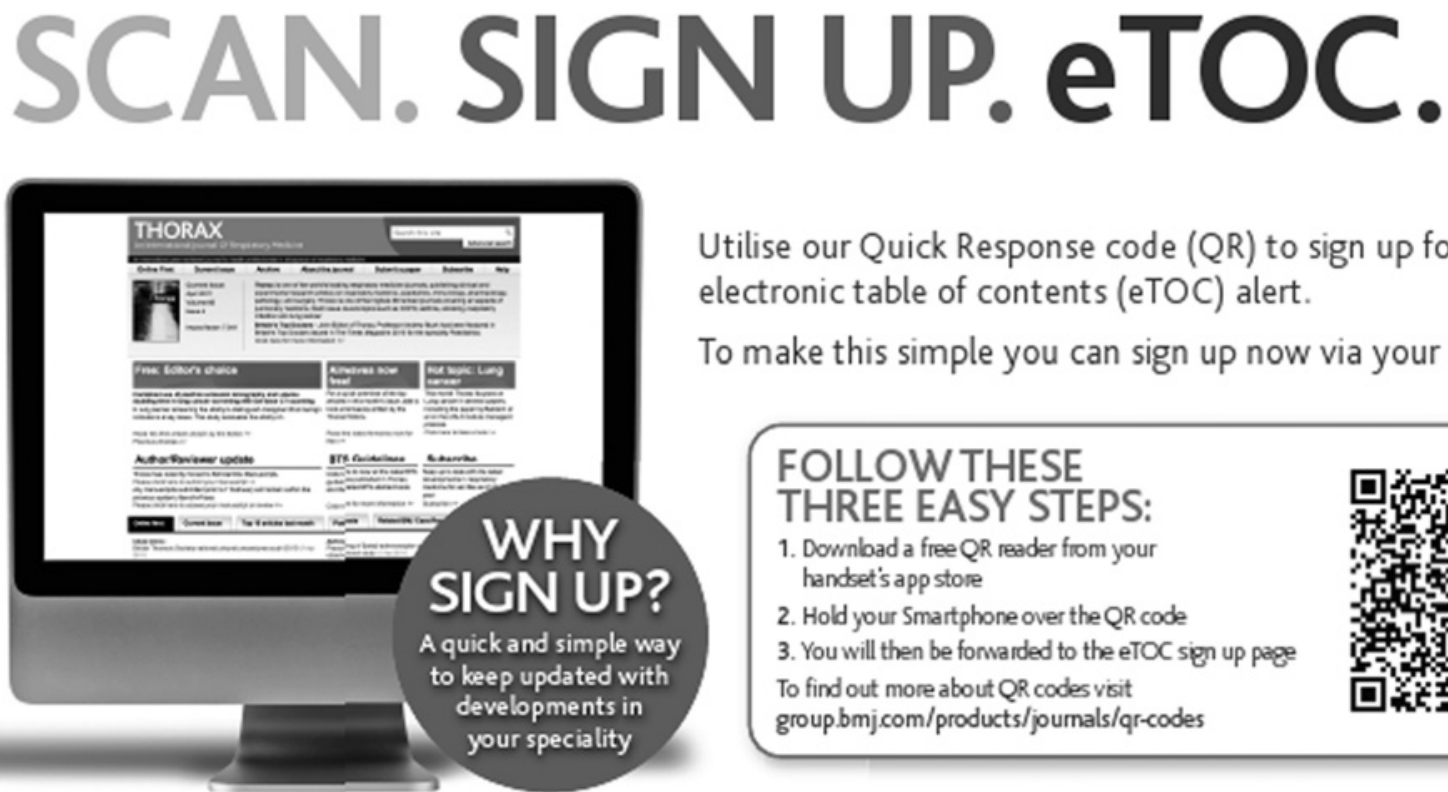

Utilise our Quick Response code (QR) to sign up for our electronic table of contents (eTOC) alert.

To make this simple you can sign up now via your Smartphone.

thorax.bmj.com

\section{FOLLOW THESE}

THREE EASY STEPS:

1. Download a free $Q R$ reader from your handset's app store

2. Hold your Smartphone over the QR code

3. You will then be forwarded to the eTOC sign up page

To find out more about $Q R$ codes visit

group.bmj.com/products/journals/qr-codes

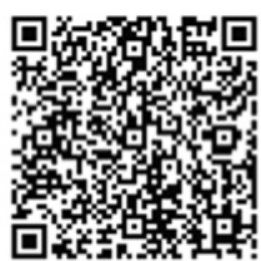

BMJIJournals 


\section{Corrections}

SV Kemp, SH El Batrawy, RN Harrison, et al. Learning curves for endobronchial ultrasound using cusum analysis. Thorax 2010;65:534-8. doi:10.1136/thoraxjnl-2009-127274

SH El Batrawy's affiliation is to read: Respiratory Medicine Department, Ain Shams University, Cairo, Egypt.

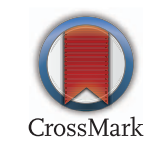

Thorax 2014;69:672. doi:10.1136/thoraxjnl-2009-127274corr1 